\title{
Blind Signal Separation Algorithm for Space-based ADS-B
}

\author{
Kai Liu ${ }^{a}$, Tao Zhang ${ }^{b}$ and Yang Ding ${ }^{c}$ \\ School of Electronic and Information Engineering, Beihang University, \\ Beijing Laboratory for General Aviation Technology, \\ Beijing Key Laboratory for Network-based Cooperative Air Traffic Management, \\ Beijing 100191, China \\ aliukai@buaa.edu.cn, bzhtao@buaa.edu.cn, cdingyang@buaa.edu.cn
}

Keywords: ADS-B, Signal Separation, Signal Collision

\begin{abstract}
Automatic Dependent Surveillance Broadcast (ADS-B) is a main technology for Air Traffic Management (ATM). As a trend of aerial surveillance, space-based ADS-B uses satellite to receive ADS-B signals to realize aerial surveillance globally. The space-based ADS-B has a series of new features that Terrestrial-based doesn't have including wider coverage, lower SNR, less power difference between signals. These new features make ADS-B signals collisions at the receiver more severe. This paper proposes a model of ADS-B signals collision pattern at the satellite based on poisson process model. This model makes the separation algorithm focus on several collision situations. This paper proposes the iterative projecting algorithm (IPA). IPA uses iteration and projecting algorithm which has less computation complexity and is more suitable for the hardware to perform. Through simulation IPA has similar performance but much lower complexity compared with other ADS-B BSS algorithms in satellite channel.
\end{abstract}

\section{Introduction}

ADS-B is a proven technique that is used in aviation management and surveillance. Although there are several links associated with ADS-B, 1090 Extended Squitter (1090ES) is the most common equipage, particularly amongst airline operators and others that fly above 18,000 ft. [1]. Terrestrialbased ADS-B technique faces the signal collision problem because this system is a burst communication system that doesn't have time slots allocation. When two or more signals overlap at the receiver antenna, demodulated module will generate large error codes. This will reduce system efficiency. Terrestrial-based system has a relatively limited coverage about $200 \mathrm{~nm}$, so the signals collision is a low probability event. Moreover the distance from different planes to the same ground station has a distinct difference so signals colliding at the receiver have a distinct power difference. A lower power signal will scarcely impact the signal with higher power level because of the receiver's dynamic minimum triggering level mechanism [2].

Space-based ADS-B has obvious difference with terrestrial-based system. It remains the equipment on the planes but utilizes LEO satellites to receive 1090ES signals. Satellite coverage area contains a large amount of planes (e.g. Iridium Next equipped with ADS-B receivers support more than 3000 planes in a coverage area). Collisions of signals are quite severe in this situation. This paper will investigate the relationship between the amounts of planes with the collision pattern. An overlapping model is proposed to make the separation algorithm focus more on the main overlapping situation. Different planes in the satellite coverage almost have the same distance to the satellite so it is hardly to pick signal out using the power difference feature like ground receive station. Furthermore small difference of direction of arrival (DOA) of signals and the limited computation performance of satellite platform also restrict the separation algorithm efficiency.

To solve the above problem, 1090ES signals or SSR mode S signals separation algorithm based on antenna array has been deeply investigated. Reference [3] proposed several algebraic methods such as MDA, AZCMA. These algorithms utilize different signal features and result in a matrix joint diagonalization problem. FastICA is proposed in [5] and has a better rate of convergence than traditional ICA. Reference [6] proposed PA and its enhanced algorithm EPA which results in Gram- 
Schmidt orthogonality. In this paper we proposed an algorithm based on iterative projecting which has similar separation efficiency but the algorithm complexity is much lower than the algorithm above and this algorithm has a significant feature that it has a structure easily implemented by hardware.

This paper is structured as follows. Section 2 introduces the collision pattern and its derivational process. Section 3 presents the signal model and IPA method. Section 4 compares the performance of IPA with MDA and EPA in simulation.

\section{Collision Pattern}

\subsection{Reception Model}

Reference [7] specifies that 1090ES signal has a duration of $120 \mu$ s and each plane updates ADS$\mathrm{B}$ messages at a nearly certain frequency. We define this frequency $M F$ which generally ranges from 1 to 2 messages per second. The time at which planes emit signals obeys uniform distribution. Fig. 1 shows a schematic view of the signals at receiver. Assume that there are $N p$ planes in the satellite coverage.

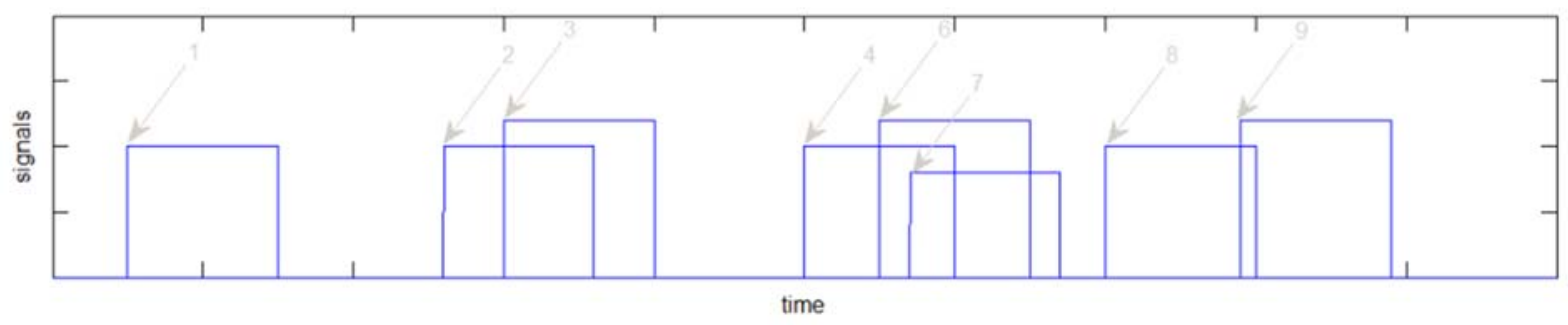

Fig. 1 Signals received schematic view

Assume $M(t)$ is the number of received signals at the receiver from an observing time point. $M(t)$ is a stochastic process. As time increase $M(t)$ counts. Assume $t 1, t 2$ are two observing point. $t 2>t 1>0$. For any time interval $\Delta t>0$ random variables $M(t 2)-M(t 1)$ and $M(t 2+\Delta t)-M(t 1+\Delta t)$ have the same probability distribution and statistic independence. So this process can be modeled by passion process.

According to passion process theory the random variable interval time $T$ between adjacent two signals has the probability distribution function

$$
F(z)=\left\{\begin{array}{cc}
1-\exp (-\lambda z) & z \geq 0 \\
0 & z<0
\end{array}\right.
$$

$\lambda$ is intensity of the passion process. $M(t)$ has the probability distribution.

$$
P\left[M\left(t_{0}+t\right)-M\left(t_{0}\right)=k\right]=\frac{(\lambda t)^{k}}{k !} \exp (-\lambda t) \quad k=1,2, \ldots
$$

$t_{0}$ is a time start point. $k$ is the number of arrival event. From (2) the expectation of $M$ in the time interval $t$ is $\lambda t$. In the space-based ADS-B situation the expectation of the amount of ADS-B messages is $N p \times M F$ per second. So $\lambda=N p \times M F$.

\subsection{Collision Pattern}

The zero order overlap is defined as the situation that one signal doesn't overlap with the forward signal nor the backward signal. One order overlap is defined as the situation that two signals overlap but neither the forward nor the backward signals overlap with the two overlapping signals show in Fig.2. $n$ order overlap is defined as the situation that $n$ signals overlap with each other but neither the forward nor the backward signals overlap with the $n$ overlapping signals. Utilize $O n$ to represent this situation. 


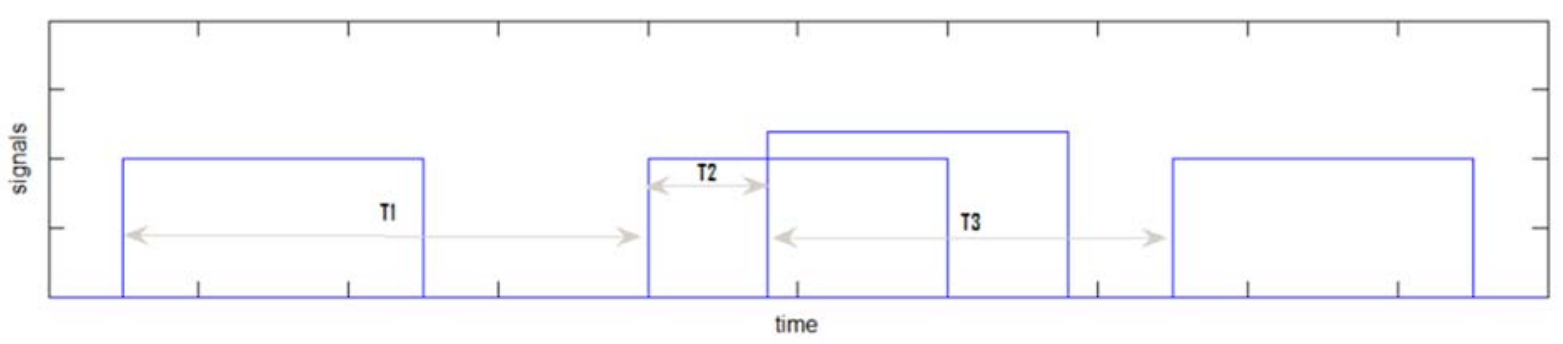

Fig. 2 One order overlap situation

Take $O 1$ situation for instance as shown in Fig.2. T1, T2, T3 is three independent random variables and obey the distribution described by (1). $O 1$ situation occurs when $T 1>120 \mu s, T 2<120 \mu s$ and $T 3>$ $120 \mu \mathrm{s}$.

This probability is $(1-F(120 \mu s))^{2} \times F(120 \mu s)$. Since the signal may overlap with its forward signal or its backward signal. This probability need to multiply by 2 . It is $(1-F(120 \mu s))^{2} \times F(120 \mu s) \times 2$. On situation occurs when $n$ signals overlap and these overlapping signals don't overlap with its forward and backward signal. This probability is $P(O n)=(1-F(120 \mu s))^{2} \times F(120 \mu s)^{n} \times(n+1)$.

Consider a common situation that $M F=1$. Np ranges from 1000 to 6000 . The collision pattern is shown in Fig.3. It shows that when planes in the satellite coverage are more than 3000 the separation algorithm must deal well with the high order overlap situation such as $O 3$ and $O 4$.

To confirm this model the simulation of collision pattern is conducted. The model agrees well with the simulation result. Details simulation results are shown in Table 1.

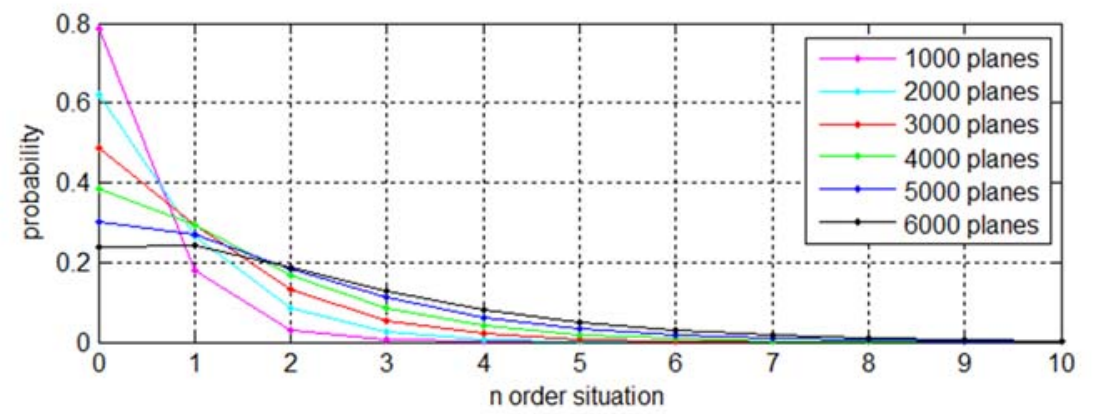

Fig. 3 collision pattern of different coverage area planes' amount.

\section{Iterative Projecting Algorithm}

\subsection{Signal Model}

Reference [3] has complete 1090ES signal model description. 1090ES signals is downconverted to middle frequency and sampled at rate $1 / T_{s}$. Single independent source data is described as

$s[n]=b[n] \exp \left(j 2 \pi n f T_{s}\right)$

$b[n]$ is the code element sequence. $f$ is the residual frequency. $d$ independent 1090ES source signals impinge on an m-element antenna array. The output signal is an $m$ rows matrix.

$\mathbf{X}=\mathbf{M S}+\mathbf{N}$

$\mathbf{X}$ is a $m \times N$ matrix. $\mathrm{m}$ rows present $\mathrm{m}$ output of the array antenna. $\mathbf{S}$ is the $d \times N$ matrix. $d$ rows present $d$ independent source signals. $N$ is the number of sample points. $\mathbf{M}$ is an unknown $m \times d$ mixing matrix which presents the array antenna responses and array signatures. $\mathbf{N}$ is an equivalent noise matrix. This is a typical array signal process equation. The accurate antenna response is not necessary but the antenna parameters will affect the algorithm performance. This will be present in simulation chapter.

Assume the antenna is a linear array where $\Delta_{k}$ is the distance between the $k$ th element and the first element $(1 \leq k \leq m)$ and $f i$ the frequency of the $i$ th source. $\mathbf{M}=\mathbf{A G}$. A is a $m \times d$ steering matrix that contains $\mathrm{m}$ vectors $\mathbf{a}\left(\theta_{\mathrm{i}}\right), 1 \leq i \leq d . \mathbf{G}$ is a $d \times d$ diagonal matrix. 


$$
\mathbf{a}\left(\theta_{i}\right)=\left[\begin{array}{c}
1 \\
\exp \left(j \frac{2 \pi f_{i}}{c} \Delta_{2} \sin \left(\theta_{i}\right)\right) \\
\vdots \\
\exp \left(j \frac{2 \pi f_{i}}{c} \Delta_{m} \sin \left(\theta_{i}\right)\right)
\end{array}\right]
$$

\subsection{Iterative Projecting Process}

In $O 2$ situation received signals $\mathbf{X}$ schematic diagram is show in Fig.4. It can be divided into several segments $\left(t_{1}, t_{2}\right),\left(t_{2}, t_{3}\right),\left(t_{3}, t_{4}\right),\left(t_{4}, t_{5}\right),\left(t_{5}, t_{6}\right)$. Each segment has a certain number of signals. Take $O 2$ for instance, IPA first utilize $\left(t_{1}, t_{2}\right)$ to estimate part of array antenna parameters and eliminate the first signal's effect. Then this overlap signals become an $O 1$ situation. Repeat the same process until the last signal remains. This is the first output signal which is not polluted by other signals. This process is called "SourceEstimate". When the last signal is estimated use ( $\left.t_{5}, t_{6}\right)$ to eliminate the last signal and then it becomes an $O 1$ situation. This process is called "OrderReduce". Manage this $O 1$ signals as above until all signals are separated.

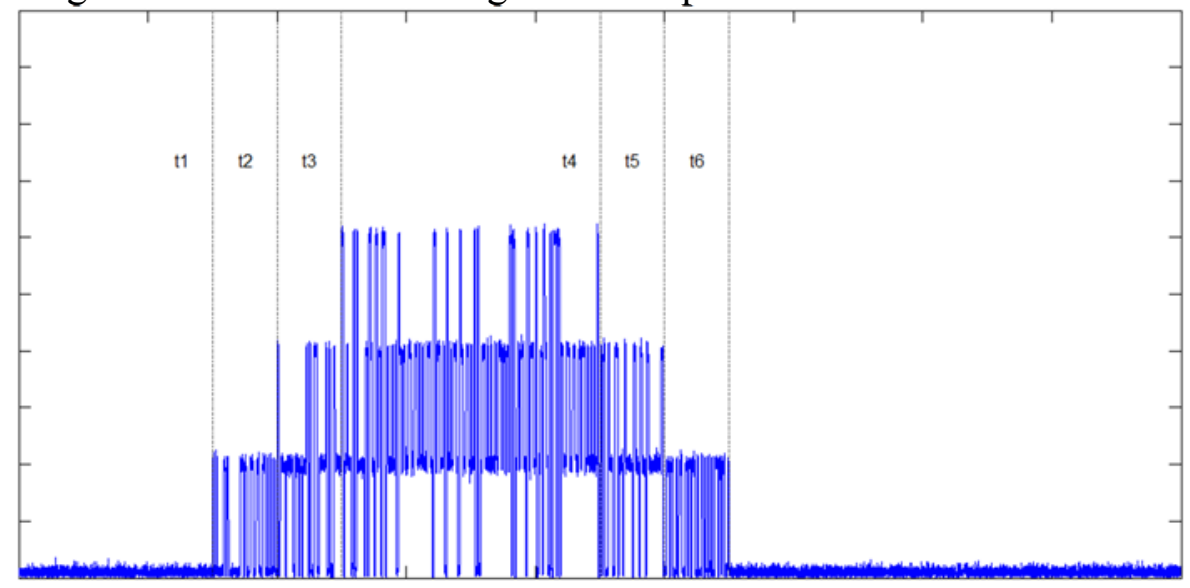

Fig. $4 \mathrm{O} 2$ situation division

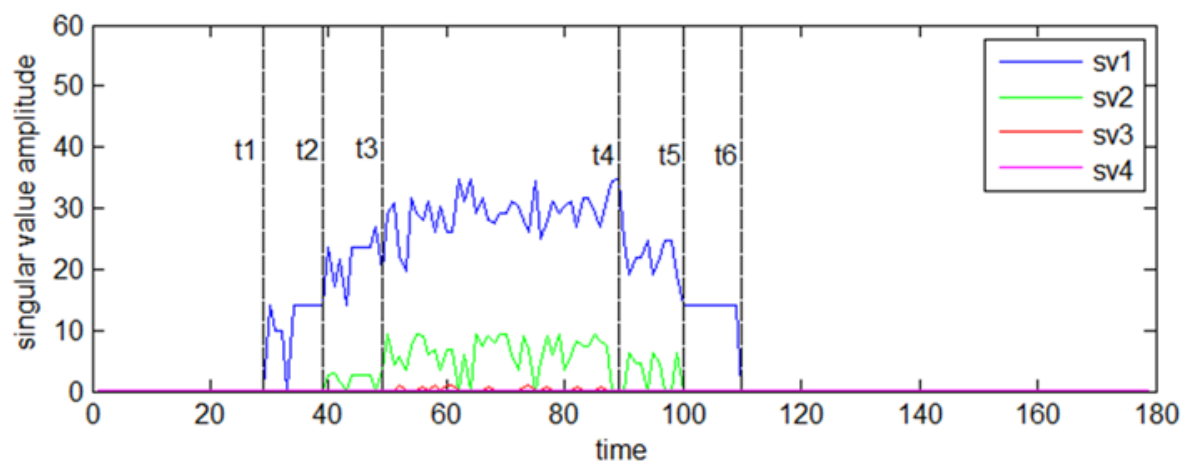

Fig. 5 Singular value in each slide window

Segment division is complemented using whiteness test algorithm such as singular value decomposition. Use a slide window with duration of $4 \mu$ s to divide the received signal and in each window a SVD is conducted. Fig. 5 shows this process.

In $\left(t_{1}, t_{2}\right)$ segment there is only one signal. Equation (4) in this segment has a simple form (6). $\mathbf{X}_{\mathbf{1}}$ is the a segment data of $\mathbf{X}$ in $\left(t_{1}, t_{2}\right)$. It is a one rank matrix so $\mathbf{m}_{1}$ can be estimated using the rank one decomposition. It is the same process to estimate $\mathbf{m}_{\boldsymbol{d}}$. 


$$
\begin{aligned}
\mathbf{X}_{(1)} & =\mathbf{M S}+\mathbf{N}=\left[\mathbf{m}_{1}, \mathbf{m}_{2}, \ldots, \mathbf{m}_{d}\right]\left[\begin{array}{c}
\mathbf{s}_{1} \\
0 \\
\vdots \\
0
\end{array}\right]+\mathbf{N} \\
& =\mathbf{m}_{1} \mathbf{s}_{1}+\mathbf{N}
\end{aligned}
$$

Once $\mathbf{m}_{\mathbf{1}}$ is estimated construct matrix $\mathbf{P}$. Each of column vectors is orthogonal with $\mathbf{m}_{\mathbf{1}}$ and has a Euclidean norm of $1 . \mathbf{w}_{\mathbf{i}} \mathbf{H}^{\mathbf{H}} \mathbf{m}_{\mathbf{1}}=\mathbf{0}$. Equation (8) explains the "OrderReduce" process.

$$
\begin{aligned}
\mathbf{P} & =\left[\mathbf{w}_{1}, \mathbf{w}_{2}, \ldots, \mathbf{w}_{d-1}\right] \\
\mathbf{X}^{\prime} & =\mathbf{P}^{H} \mathbf{X}=\mathbf{P}^{H}(\mathbf{M S}+\mathbf{N}) \\
& =\left[\begin{array}{c}
\mathbf{w}_{1}^{H} \\
\mathbf{w}_{2}^{H} \\
\vdots \\
\mathbf{w}_{m-1}^{H}
\end{array}\right]\left[\begin{array}{llll}
\mathbf{m}_{1} & \mathbf{m}_{2} & \ldots & \mathbf{m}_{d}
\end{array}\right]\left[\begin{array}{c}
\mathbf{s}_{1} \\
\mathbf{s}_{2} \\
\vdots \\
\mathbf{s}_{d}
\end{array}\right]+\mathbf{N}^{\prime} \\
& =\left[\begin{array}{cccc}
\mathbf{0} & \mathbf{w}_{1}^{H} \mathbf{m}_{2} & \cdots & \mathbf{w}_{1}^{H} \mathbf{m}_{d} \\
\mathbf{0} & \mathbf{w}_{2}^{H} \mathbf{m}_{2} & \vdots & \mathbf{w}_{2}^{H} \mathbf{m}_{d} \\
\vdots & \vdots & \vdots & \vdots \\
\mathbf{0} & \mathbf{w}_{m-1}^{H} \mathbf{m}_{2} & \cdots & \mathbf{w}_{m-1}^{H} \mathbf{m}_{d}
\end{array}\right]\left[\begin{array}{c}
\mathbf{s}_{1} \\
\mathbf{s}_{2} \\
\vdots \\
\mathbf{s}_{d}
\end{array}\right]+\mathbf{N}^{\prime} \\
& =\left[\begin{array}{cccc}
\mathbf{0} & \mathbf{m}_{1}^{\prime} & \ldots & \mathbf{m}_{d-1}^{\prime}
\end{array}\right]\left[\begin{array}{c}
\mathbf{s}_{1} \\
\mathbf{s}_{2} \\
\vdots \\
\mathbf{s}_{d}
\end{array}\right]+\mathbf{N}^{\prime} \\
& =\mathbf{M}^{\prime}+\mathbf{N}^{\prime}
\end{aligned}
$$

New matrix $\mathbf{X}^{\prime}$ has $(m-1)$ rows. Each of the rows represents a new antenna array output. "SourceEstimate" process uses $(8)(d-1)$ times to estimate the last signal relative to the input signals. "OrderReduce" process uses this process one time to eliminate the last signal relatively to the input signals. $\mathbf{M}$ ' is a new $m \times(d-1)$ antenna response matrix. $\mathbf{S}$ ' is a (d-1) rows new source matrix which only contains $\mathbf{S}_{2}$ to $\mathbf{S}_{\mathrm{d}}$. $\mathbf{N}^{\prime}$ is a new equivalent noise matrix. The elimination of $\mathbf{S}_{\mathbf{d}}$ has the same process with $\mathbf{s} 1$.Separation of an $O 2$ situation is shown in simulations and results chapter Fig.7

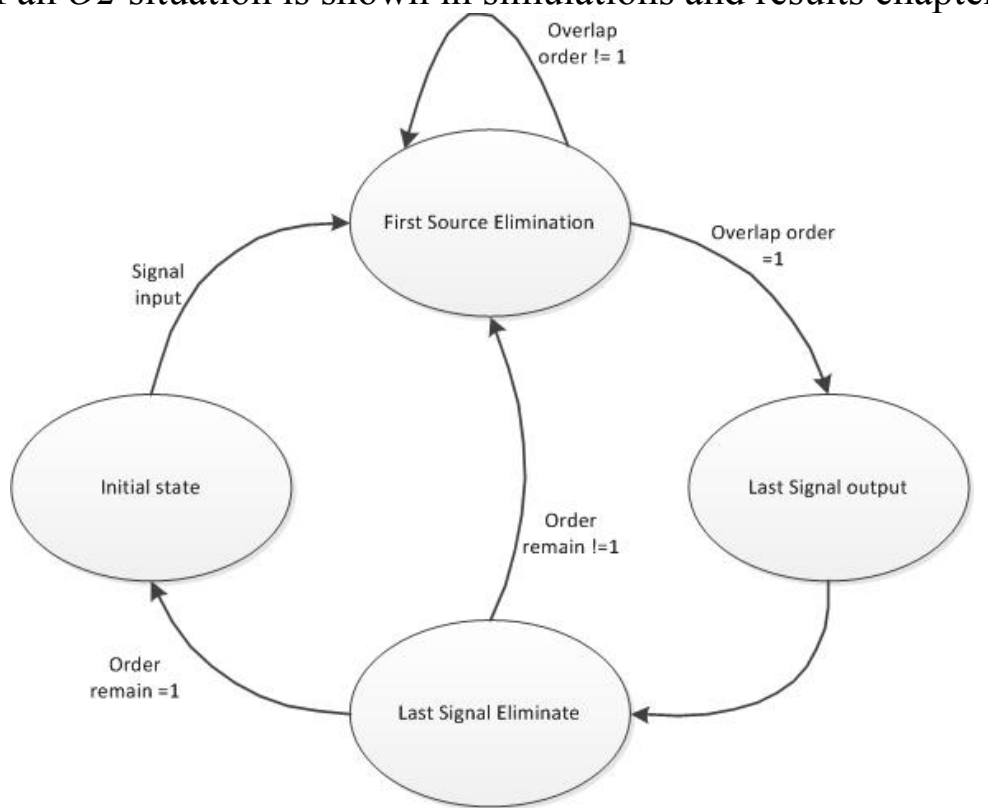

Fig. 6 IPA state transition diagram 
This algorithm is very suitable for hardware implementation. Its structure contains three main computation part - singular value computation, $\mathbf{P}$ matrix construction, and matrix multiplication. An accurate flow control is also needed but it can be easily complemented by hardware using state machine. The state transition diagram is shown in Fig.6.

\section{Simulation and Results}

\subsection{Collision Pattern Simulation}

Collision pattern model simulation uses $N$ variables to simulate planes. Each variable generates a time point in uniform distribution at $0 \sim 1 \mathrm{~s}$ to simulate the emitting of 1090ES signals. Use a statistical program to obtain the collision distribution. Each simulation has 5000 times' random repetition for relatively accurate statistic values. Results are shown in Table 1.

Table 1 Probability comparison of theoretical values and experimental values

\begin{tabular}{ccccccc}
\hline & \multicolumn{2}{c}{$\boldsymbol{N}=\mathbf{2 0 0 0}$} & \multicolumn{2}{c}{$\boldsymbol{N}=\mathbf{4 0 0 0}$} & \multicolumn{2}{c}{$\boldsymbol{N}=\mathbf{6 0 0 0}$} \\
\hline & $\mathbf{T V}$ & $\boldsymbol{E} \boldsymbol{V}$ & $\mathbf{T V}$ & $\boldsymbol{E} \boldsymbol{V}$ & $\boldsymbol{T V}$ & $\boldsymbol{E V}$ \\
O0 & 0.6188 & 0.6188 & 0.3829 & 0.3824 & 0.2369 & 0.2368 \\
O1 & 0.2641 & 0.2632 & 0.2919 & 0.2917 & 0.2432 & 0.2430 \\
O2 & 0.0845 & 0.0843 & 0.1669 & 0.1666 & 0.1872 & 0.1871 \\
O3 & 0.0240 & 0.0238 & 0.0849 & 0.0849 & 0.1281 & 0.1280 \\
O4 & 0.0064 & 0.0064 & 0.0404 & 0.0405 & 0.0822 & 0.0823 \\
\hline
\end{tabular}

\subsection{IPA Process and Performance}

Fig.7.a shows the process of separation of an $\mathrm{O} 2$ situation. Fig.7.a c shows the "SourceEstimate" process. Fig.7.c is the estimated output of the last signal. Fig.7.d is detail of Fig.7.c. Fig.7.e is the "OrderReduce" process. IPA uses the unpolluted signal at the end of the overlapping signals to eliminate the effect of the last signal.

In $O 2$ situation with the same antenna parameters the comparison of three algorithms-MDA, EPA and IPA is conducted. Using a traditional method to decode the separated signals and compared with the original source the BER figure is shown in Fig.8. EPA and IPA have similar performance and MDA is weaker. SNR of separated signal is a norm of the separation algorithm's performance. In the same situation the IPA and EPA have similar output SNR and MDA is lower as the Fig.9 shows. Fig.10 shows the elapsed time for algorithm conducting for different overlap situations. The computation platform is MATLAB R2013a. When the order of overlap increases the IPA has the least conduct time than the other two algorithms. IPA algorithm complexity is proportional to the order $\mathrm{n}$ and EPA algorithm complexity is proportional to the $\mathrm{n}^{2}$ in estimation.

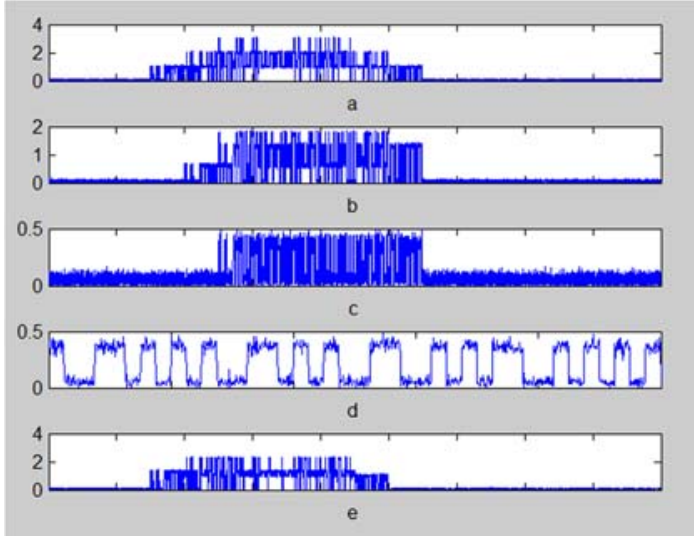

Fig. 7 IPA process

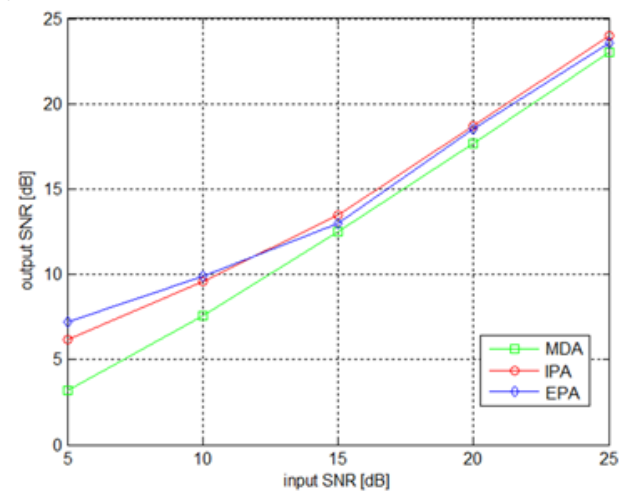

Fig. 8 Comparison of output SNR of separation algorithm 


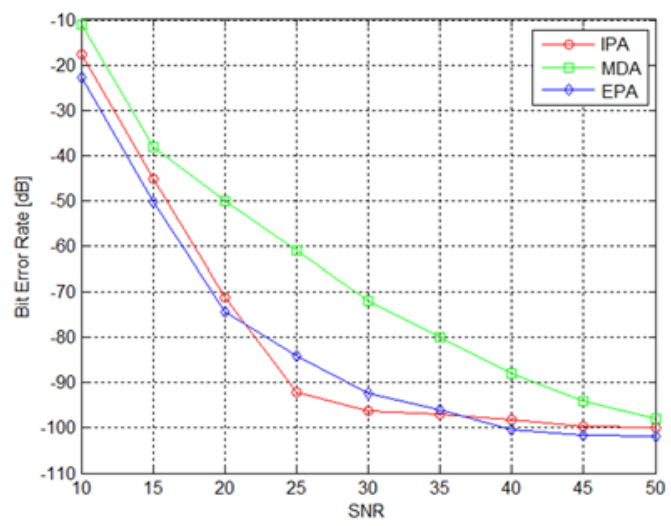

Fig. 9 BER performance comparison

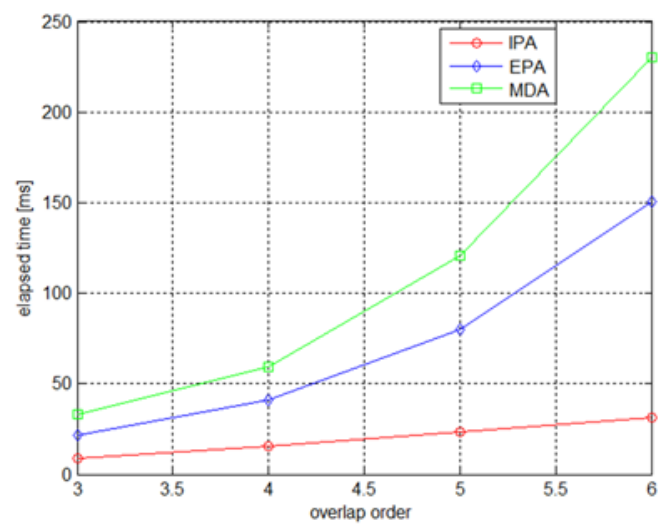

Fig. 10 Algorithm complexity comparison

\section{Summary}

In this paper we presented the blind ADS-B signal separation called IPA for satellite condition. The collision pattern in satellite condition shows that 3 order and 4 order overlap situation cannot be ignored. The separation algorithm needs to fit to high order overlap situations. IPA has a lower computation complexity than other algorithms and has a structure that is easy to implement for hardware. In space-based ADS-B it is a suitable method for the signal collision problem.

\section{References}

[1]. M. A. Garcia, J. Stafford, J. Minnix and J. Dolan, "Aireon space based ADS-B performance model," Integrated Communication, Navigation, and Surveillance Conference (ICNS), 2015, Herdon, VA, 2015, pp. C2-1-C2-10.

[2]. W. Harman, J. Gertz and A. Kaminsky, "Techniques for improved reception of $1090 \mathrm{MHz}$ ADSB signals," Digital Avionics Systems Conference, 1998. Proceedings., 17th DASC. The AIAA/IEEE/SAE, Bellevue, WA, 1998, pp. G25/1-G25/9 vol.2.

[3]. N. Petrochilos and A. J. van der Veen, "Algebraic Algorithms to Separate Overlapping Secondary Surveillance Radar Replies," in IEEE Transactions on Signal Processing, vol. 55, no. 7, pp. 37463759, July 2007.

[4]. G. Galati, N. Petrochilos and E. G. Piracci, "Degarbling Mode S replies received in single channel stations with a digital incremental improvement," in IET Radar, Sonar \& Navigation, vol. 9, no. 6, pp. 681-691, 72015.

[5]. YAN Ke-yi, LYU Ze-jun and SHI Hong-wei, “An improved FastICA ADS-B 1090ES Signal Separation Technique," in JISUANJI YU XIANDAIHUA 2014.

[6]. N. Petrochilos, G. Galati and E. Piracci, "Separation of SSR Signals by Array Processing in Multilateration Systems," in IEEE Transactions on Aerospace and Electronic Systems, vol. 45, no. 3, pp. 965-982, July 2009 ..

[7]. RTCA DO-260B,"Minimum Operational Performance Standards for $1090 \mathrm{MHz}$ Extended Squitter Automatic Dependent Surveillance-Broardcast (ADS-B) and Traffic information Services-Broadcast (TIS-B), ’December 2,2009 